\title{
Tuberkulose-Therapie nach Dr. Carl Spengler mit besonderer Berücksichtigung des I.K. \\ Von
}

\author{
Dr. R. Benöhr, \\ Assistent von Dr. C. Spengler. \\ Mit 1 Kurventafel.
}

Durch die Immunkörper (I.K.)-Therapie und ihre Einführung in die Praxis hat uns Dr. Carl Spengler ein wirksames und zuverlässiges Mittel zur Bekämpfung der Tuberkulose gegeben. Diese ImmunkörperTherapie hat schon - nach zahlreichen Berichten von Ärzten aus allen Ländern - eine weite Verbreitung gefunden, und die uns berichteten vielen Erfolge decken sich zum grossen Teil mit unsern guten Resultaten hier an Ort und Stelle.

Untenstehend folgt ein grösseres Krankengeschichtenmaterial Carl Spenglers und im Anhang ein solches aus der Tieflandpraxis von Dr. Pumr und Dr. Westphal.

Zunächst will ich die ganze Art und Weise der Carl Spenglerschen Tuberkulose-Therapie in ihren Grundzügen skizzieren.

\section{Diagnostik.}

Um eine wirksame Behandlung der Tuberkulose und eine kritische Beurteilung des Krankheitsverlaufs durchführen zu können, ist eine sorgfältige Erkennung der Krankheit und ihrer Verlaufsformen unerlässlich. Zur Diagnose gehört die klinische, die bakterjologische Untersuchung und die biologische Blutuntersuchung.

\section{Klinische Cntersuchung.}

Die physikalische Untersuchung der Lungen ist mit der Konstatierung der landläufigen perkutorischen und auskultatorischen Phä- 
nomene nicht erschöpft. .Um auch in Zweifelsfällen zu einer exakten Diagnose zu gelangen, müssen wir vielmehr alle uns zu Gebote stehenden Feinheiten der Untersuchungsmethodik zu Hilfe nehmen. Dazu gehört bei der physikalischen Untersuchung auch die Auskultation der Flüsterstimme, die viel vernachlässigt worden ist und erst in letzter Zeit wieder mehr befürwortet wird (Moses, M asing). Spengler hat ihren Wert seit langem erkannt und der Auskultation der Flüsterstimme bei seinen Untersuchungen einen festen Platz angewiesen. Als Frühsymptom bei der Erkrankung, wie Moses es angibt, und als Finalsymptom bei der Heilung, wie Masing dem hinzufügt, haben wir die Broncholoquie in vielen Fällen als einzigen Anhaltspunkt für die Erkrankung gefunden, wo nicht einmal verschärftes Atmen zu hören war. In seiner ausführlichen Abhandlung bespricht Moses die physikalischen Bedingungen beim Zustandekommen der Bronchophonie und gibt die Erklärung, dass die durch Resonanz in den Bronchien verstärkte Stimme durch das leitungsfähige luftleere Lungengewebe nach der Brustwand geleitet wird, deren Vibrationskraft ihrerseits durch die Erkrankung noch erhöht sein kann.

Noch prägnanter ist die Auskultation der Flüsterstimme über den Kavernen, das von Carl Spengler "Cavernoloquie" genannte Symptom. Wir lassen, wie bei der Bronchophonie, die Patienten mit leiser Flüsterstimme das Wort "99" oder die-Russen "Petersburg" aussprechen. Der Patient spricht gewissermassen in das Stethoskop hinein. In Verbindung mit den anderen Hilfsmitteln ist dies ein gutes Diagnostikum zum genauen Diagnostizieren von Vorhandensein, Lage und Umfang von Kavernen. Man kann die Kaverne genau abgrenzen, wenn man sich unter Auskultation der Flüsterstimme mit dem Stethoskop auf der Brustwand der Kaverne nähert oder entfernt. Ein weiteres Symptom zur Erkennung von Hohlräumen, das bisher noch nicht veröffentlicht worden ist, ist das von Spengler angegebene "A nblasegeräusch". Man hört dieses Geräusch über der Kaverne, wenn - man den Patienten ein kurzes kräftiges stimmloses „h" ausstossen lässt. Die Kaverne muss. mit einem Bronchus in Zusammenhang stehen, dann gibt das Anblasen der Luft in der Kaverne ein charakteristisches metallisch-schwirrendes Geräusch. Dies Phänomen gilt nur für den Oberlappen, da die Luftzufuhr von unten kommen muss.

Erwähnen will ich auch an dieser Stelle die vergleichende Palpation des Thorax, wie sie Pottenger angegeben hat: die kranke Lungenseite gibt sich durch eine vermehrte palpatorische Resistenz zu erkennen. 
Von der weiteren klinischen Untersuchung will ich hier nur die Temperaturmessung anführen. Im allgemeinen lassen wir unsere $\mathrm{Pa}$ tienten, soweit keine Kontraindikation vorliegt, die Messungen im Munde vornehmen. Der Begriff des "Fiebers", wie ihn die Physiologen fassen - s. Landois, Physiologie, n38-390 leichtes Fieber, $39-41^{0}$ schweres Fieber" - ist für den Kliniker nicht brauchbar, und es wäre für den Kliniker fehlerhaft, Temperaturen bis 37,5 als normal anzusehen. Als Normaltemperatur betrachten wir eine nicht über $57^{\circ}$ (Mundmessung, in der Ruhe gemessen) hinausgehende Körperwärme, deren Tagesschwankungen einen halben Grad nicht überschreiten. Bei der Immunkurve finden wir überhaupt nur sehr geringe Tagesschwankungen von $1 / 10-3 / 10^{0}$ (Beispiele siehe im zweiten Teil unter den Kurven).

\section{Bakteriologische Untersuchung.}

Eine wesentliche, unentbehrliche Vervollständigung des Bildes, das man aus der klinischen Untersuchung gewonnen hat, ist eine exakte Analyse des Sputums, sowie eventuell anderer Se- und Exkrete der Kranken. Zur Sputumanalyse gehört vor allem die 24stïndige Sputum-Messung, die Beobachtung der vorhandenen Zellarten, der Nachweis der verschiedenen Tuberkuloseerreger, ihrer Gestalt, Verteilung und Zahl, eventuell auch deren Lebensfähigkeit in der Kultur und der tierexperimentell festzustellenden Virulenz.

In dem Spenglerschen Institut wird nach folgenden von ihm angegebenen Methoden diese Untersuchung gehandhabt:

1. Pikrinfärbung.

2. Hüllenmethode.

3. Zi ehl-Spengler-Färbung.

4. Phagozytenfärbung.

5. Pankreatinverdauung zur Sedimentierung der Tuberkelbazillen.

6. Anreicherungsmethode und Züchtungen ${ }^{1}$ ).

Zur gleichmässigen und vergleichsfähigen mikroskopischen SputumUntersuchung ist die Homogenisierung des Sputums auf dem Deckglas das zweckmässigste und einfachste Verfahren. Man reisst von verschiedenen Sputum-Ballen kleine Partikel mit einem Platinhaken ab und mischt sie auf dem Deckglas oder Objektträger homogenisiert - färbt ein geeignetes Quantum und erbält in dieser Weise zuverlässige Durchschnitts- und Vergleichswerte.

ad 1. Pikrinmeth od $\mathrm{e}^{8}$ (Karbolfuchsin erwärmen in der Flamme,

1) Anmerkung bei der Korrektur: Inzwischen ist aus dem Carl Spenglerschen Laboratorium noch eine neue Färbemethode angegeben: Karbolfuchsin-Jod von Kronberger. Siehe Beitr. z. Klin. der Tub. Bd. XVI. H. 2. 
bis leichte Dämpfe aufsteigen; Pikrinsäure kurz darauf giessen, Entfärben mit $15 \%$ Salpetersäure und $60 \%$ Alkohol, Nachfürben mit Pikrinsäure). Diese Methode ist die zweckmässigste zur gewöhnlichen Sputumfärbung. Sie färbt sämtliche vorhandenen Bazillen und Splitter. Ihre Überlegenheit über die gewöhnliche $\mathrm{Ziehlfärbung} \mathrm{lässt} \mathrm{sich}$ durch Kontrollpräparate leicht beweisen: häufig zeigt uns das Pikrinpräparat Bazillen, die mit $\mathrm{Z}$ i ehlfärbung nicht nachgewiesen werden können. Auch die Morphologie der Bazillen ist hierbei gut zu erkennen; wir sehen, ob die Bazillen gut färbbar sind, ob sie ganz sind oder hüllengeschädigt.

ad 2. Hüllenmethode ${ }^{8}$. Sie dient, wie die Pikrin-Methode, ebenfalls zur Unterscheidung von. Tuberkelbazillen und HumanoLongus; die letzteren ersçheinen durch diese Methode weit grösser als die ersteren.

ad 3. Ziehl-Methode, modifiziert nach Spengler ist gut zum Nachweis der Zellbestandteile und eventuell Bakterien-Einschlüsse, ferner der Schollenkerne und luetischen Maulbeerzellen.

ad 4. Phagozytenfärbung. Sie färbt den Kern der Zellen blaurot, das Protoplasma hellgrün und die Bakterien rot, und gibt uns ein gutes Bild über die eventuelle Phagozytose und den Chemotropismus. Wir haben durch sie eine gewisse Kontrolle der Wirkung der spezifischen Behandlung, ähnlich wie bei der Hüllenschädigung.

Für gewöhnliche Sputum-Untersuchungen brauchen wir meist mehrere dieser Methoden, je nach dem Zweck der mikroskopischen Untersuchung. In allen negativen Sputumanalysen aber wenden wir alle Methoden an und fügen auch noch die Antiformin- und Antiformin-Ligroinmethode hinzu.

Nicht vernachlässigen darf man die Urinuntersuchungen, wie dies oft der Fall zu sein scheint. Häufig konnten wir bei $\mathrm{Pa}$ tienten, noch bevor klinische Symptome einer Nephritis auftraten, Bazillen oder Splitter im Urin nachweisen, auch im eiweissfreien Urin. Zur Unterscheidung von den Smegmabazillen dient die Perlsuchtkaltfärbung mit Voralkalisierung und die Formalinkultur oder die Waschzüchtung aus Flöckchen. Der positive Bazillenbefund im Stuhl ist ein viel häufigerer, als man annimmt, im diarrhöischen sowohl wie im Obstipationsstuhl. Auf die Häufigkeit der tuberkulösen Darmerkrankung weist auch $\mathrm{Hollos}$ hin in seiner ausgezeichneten Abhandlung: "Die tuberkulösen Intoxikationen."

Der regelmässige Nachweis von Tuberkuloseerregern in grösserer Zahl im Stuhl beweist eine tuberkulöse Darmaffektion. Gelegentliches Auftreten spärlicher Bazillen im Stuhl kommt ror, wenn Sputum morgens nüchtern in grösseren Mengen verschluckt wird. 
Ausser den oben besprochenen Färbemethoden stehen uns natürlich Kulturen und Tierversuche zu Gebote.

Zur Erkennung der Bakterien der Sekundärinfektion wird der steril aufgefangene Sputumballen nach der S penglerschen Kernwaschung und Sedimentierung untersucht, oder mit den Methoden nach Koch-Kitasato und Pfeiffer. Nach den von Spengler veröffentlichten Arbeiten liegt das Kriterium der Mischinfektion in der mechanischen Untrennbarkeit von Tuberkelbazillen und Sekundär-Bakterien; der Sputumkern enthält beide Arten nebeneinander. Die Begleitinfektion dagegen ist die chronische Bronchitis der Lungentuberkulösen; der Sputumkern enthält hier nur Tuberkelbazillen, und die Sekundärbakterien finden sich ausschliesslich in den Kernumhüllungen.

\section{Blutuntersuchung.}

Schon die Hämoglobinbestimmung kann uns eine gewisse Auskunft geben über den Gehalt an Immunkörpern. Niedriger Hämoglobingehalt geht meist Hand in Hand mit niedrigem Immunkörpergehalt; eine Steigerung der Immunkörper bewirkt auch eine Steigerung des Hämoglobins und umgekehrt.

Über den Gehalt an spezifischen Agglutininen und Präzipitinen im Blut werden wir durch die Agglutinations- und PräzipitationsMethoden unterrichtet. (Näheres darüber siehe Fuchs-Wolfring: Zur C. Speng lers Blutzellenimmunität, Beitr. zur Klinik der Tuberkulose, Bd. XIV, 1909). Die Blutuntersuchung ist wertvoll für die Erkennung des Immunkörpergehalts bei Beginn, sowie für seine Kontrolle im Verlauf der Behandlung. Die Kontrolle des Hämoglobins und der Immunkörper zeigt uns auch, wie weit der Kranke hämound immunopoetisch leistungsfähig ist. So muss man bei einer Verminderung des Hämoglobins die körperlichen Leistungen (Gehen, Bewegungen) sowohl, wie das Liegen im Freien einschränken, wie überhaupt die Liegekur einer wissenschaftlichen Beobachtung unterzogen werden muss.

Schliesslich kommt bei der Diagnostik noch die Tuberkulin-Injektion in Betracht. Doch wird sie da, wo man die oben erwähnten Untersuchungen sorgfältig anwendet, nur selten nötig sein. Wenn man sie macht, soll man sich an kleine Tuberkulindosen halten und den Dualismus berücksichtigen. Letzteres geschieht am besten dadurch, dass man auf dem einen Arm ATO, auf dem anderen Arm PTO zur Anwendung bringt.

Beginn der diagnostischen Injektionen mit je 1/100-1/10 mg ATO und PTO und bis $1 \mathrm{mg}$ steigend. Schon die Differenz in der Stärke 
der Stichreaktion zeigt eine vorhandene Tuberkulose an und orientiert über das Vorwiegen der Tuberkelbazillen oder des "Humano-Longus" Carl Spenglers.

\section{Therapie.}

Wir behandeln unsere Patienten in der Ambulanz und in der Hausbehandlung. Für Kranke, die der Schwesternpflege bedürfen, haben wir hier das Alexanderhaus-Sanatorium und das Sanatorium St. Josefshaus. Leichtere Fälle können mit der spezifischen Behandlung ohne Berufsstörung geheilt werden.

Die Theorie der Immunkörper-Therapie will ich hier ganz kurz wiederholen. Carl Spengler hat nachgewiesen, dass die Hauptproduktions- und Anhäufungsstätten der Tuberkulose-Immunsubstanzen die roten Blutkörperchen sind, und gewann durch Extraktion dieser Immunkörper aus den Blutzellen bezw. durch Verwendung des aufgeschlossen gelösten Gesamtblutes gegen Tuberkulose künstlich immunisierten Blutes von Schafen und Kaninchen vornehmlich die Substanzen zur Immunisierung der Tuberkulosekranken. Das I.K. hat, seinem Gehalt an Antitoxinen und Lysinen entsprechend, eine doppelte Wirkung, eine direkt giftbindende (antitoxische) und eine bakterientötende (lytische). Die Immunkörper-Therapie ist ein passiv-aktives Verfahren, denn die Lysocidine lösen die Infektionskeime auf, so dass deren Gifte bei der Resorption zur aktiven Immunitätssteigerung herbeigezogen werden.

Wir wenden das I.K. in Form von Injektionen und Einreibungen an. Näheres über Anwendungs- und Wirkungsweise siehe unten.

Hier möchte ich nur auf die Einreibungen näher eingehen.

In den Lehrbüchern der Physiologie wird eine Resorption von flüssigen Stoffen in die Haut durch Einreibungen geleugnet. Dieser Satz bedarf einer Berichtigung, denn das Gegenteil davon ist unschwer zu beweisen.

In der Histologie wird angegeben, dass die Haut fähig ist, flüssige und gasförmige Ausscheidungsprodukte aus dem Organismus zu entfernen. Damit ist das Prinzip der Impermeabilität der Haut schon. -durchbrochen. Man hat bei den Einreibungen von medikamentösen Lösungen den Einwurf der Wirkungslosigkeit erhoben; sie drängen nicht in die Haut ein, sondern verdunsten beim Reiben auf der Haut. Dass dies nicht der Fall ist, beweist folgender Versuch: Man giesse auf eine etwas erwärmte Glasplatte einige $\mathrm{ccm}$ einer Flüssigkeit und reibe sie mit den mit wasserdichtem Stoff bedeckten Fingern. Nach langem Reiben wird man nur eine sehr geringe Verdunstung bemerken, der grössere Teil der Flüssigkeit bleibt auf der Glasplatte 
zurück. Dagegen reibe man dieselbe Menge Flüssigkeit auf die Haut (Unterarm), so wird man bemerken, dass in ganz kurzer Zeit die Flüssigkeit verrieben ist. Da sie nach obigem Versuch nicht verdunstet ist, kann sie nur in die Haut eingedrungen sein. Dass die Flüssigkeit auch wirklich in die Lymph- und Blutbahnen des Körpers gelangt, haben wir durch folgenden Versuch nachgewiesen: Nach Einreibung von 20 Tropfen $1 \%$ iger Jodkalium-Lösung auf den Unterarm konnte schon nach 20 Minuten im Speichel sowohl wie im Urin eine dentliche Jodreaktion nachgewiesen werden.

Auf diesen Tatsachen fussend, hat Spengler schon seit Jahren die Einreibungen befürwortet, schon bevor Morro die perkutane Anwendung von Tuberkulin empfahl.

In vielen Fällen, besonders bei sensiblen Patienten, ist die Einreibung ein guter Ersatz für die Injektion. Die Einreibung wirkt nicht so intensiv und nicht so schnell, verdient aber den Vorzug, wenn man bei Injektionsscheuen sichere Wirkungen haben will. Mit den I.K.-Einreibungen haben wir in gleicher Weise Immunkörpersteigerung erzielt, wie mit den Injektionen (vgl. unten Krankengeschichten). Hollos hat seine ausgezeichneten Erfolge mit I.K. grösstenteils mit Einreibungen erreicht.

Die perkutane Anwendung eignet sich auch für andere Medikamente, bei denen man aus irgend einem Grunde die Darreichung per os oder per injectionem vermeiden will: Digalen, Morphium, Codein etc. Diese Anwendungsweise hat sicherlich eine grosse $\mathrm{Zu}$ kunft. Die Wirkungen sind ausserordentlich günstig und zwar bei den verschiedensten wasserlöslichen Medikamenten. Mit Vorliebe machen wir jetzt die Einreibungen von Medikamenten über den erkrankten Körperteilen.

\section{Lungensyphilis und Tuberkulose.}

Eine wesentliche Rolle bei der tuberkulösen Erkrankung spielt die Syphilis - sei es in der Form der akquirierten oder der hereditären. Diese Tatsache ist von den meisten Tuberkulose-Ärzten viel zu wenig beachtet worden, besonders in der Neuzeit. Früher wurde von Schnitzler, Fournier, Senator, Kobert u. a. auf den Zusammenhang zwischen Lues und Tuberkulose mehrfach hingewiesen. Neuerdings betont $\mathfrak{u}$. a. S. K lein die häufige Koinzidenz zwischen Lues und Tuberkulose; er gibt etwa $41 \%$ bei einem grösseren Krankenmaterial an. Seit mehr als zwanzig Jahren hat Carl Spengler dieser Frage seine Aufmerksamkeit zugewandt, und ist nunmehr zu der Überzeugung gekommen, dass zahlreiche Tuberkulosefälle auf hereditär-luetischer Konstitution sich entwickeln. Die durch die 
Syphilisgifte geschädigten Organismen sind prädisponiert für Tuberkulose. Die Verbreitung der Lues ist sicherlich eine viel grössere, als man gemeinhin annimmt, nicht nur die hereditäre, sondern auch die erworbene. Denn die Tatsache, dass in syphilitischen Placques oft massenhaft Spirochäten sich nachweisen lassen, weist schon darauf hin, dass auch die extragenitale Verbreitung der Syphilis durch Sprechen, Husten, Niesen (Tröpfchen-Theorie) und natürlich auch durch Ess- und Trinkgeschirre möglich und sehr wahrscheinlich ist.

Die klinischen Erfahrungen einer langjährigen Tuberkulose-Therapie weisen mit zwingender Notwendigkeit auf das häufige Vorkommen der Lungensyphilis hin, wenn auch auf dem Sektionstisch der anatomische Nachweis schwer zu leisten ist. Das anatomisch-pathologische Bild der Lungensyphilis ist gewöhnlich, zumal auch bei Heredität, die "fleischige" hypertrophische Lunge, die bei länger bestehendem Prozess mit der Cirrhose sich kombinieren kann, analog der zirrhotischen Leber mit Bindegewebsinduration.

Ein charakteristisches auskultatorisches Phänomen über den "fleischigen" Lungen ist das ebenfalls von Carl Spengler angegebene Rauschen. Dem gewöhnlichen Sitz der Lungensyphilis entsprechend findet es sich häufiger über Mittellappen und Unterlappen, als über dem Oberlappen. Meistens ist das Rauschen interponiert, das heisst, es erstreckt sich nur auf die Endphase des In- und die Anfangsphase des Exspiriums.

Die Diagnose der mit Tuberkulose kombinierten Syphilis erstreckt sich weiter auf folgende Punkte:

1. Eine häufige Miterkrankung des Larynx, die oft die primäre Erkrankung ist.

2. Sind die Bronchien fast immer auffallend miterkrankt, besonders bei der acquirierten Lues. Auskultatorisch gibt sich diese luetische Bronchitis durch Bronchialsymptome und nicht selten durch ein "Schnarren" oder "Schnarchen" zu erkennen.

3. Die Improportionalität des Falles, d. h. der ausgedehnte Lungenprozess im Gegensatz zum guten Aussehen und Ernährungszustand des Patienten.

4. Das Sputum der Syphilitisch-Tuberkulösen. Hohe Sputummenge bis zu mehr als $300 \mathrm{ccm}$ ) eines schleimigen oder schleimig-eiterigen Auswurfs. Der mikroskopische Befund ist charakteristisch durch aussergewöhnlich zahlreiche zellige Elemente mit Schollenkernen, im Gegensatz zu den sonst scharf umgrenzten Zellkernen. Tuberkelbazillen finden sich meist wenig, auch bei ausgedehntem Lungenprozess. Der Nachweis von Spirochäten im Sputum gelingt zuweilen. 
Als Therapeutikum gegen die Lungensyphilis verordnet Spengler Jod und zwar innerlich als Jodeiweiss in Form der nach seinen Angaben hergestellten Jokasin-Tabletten ${ }^{1}$ ) (drei Wochen lang zum ersten Frühstück täglich eine Tablette, dann nach dreiwöchentlicher Pause wieder anfangen). Für die Armen- und Kassenpraxis kann Jodtinktur in warmer Milch an ihre Stelle treten (5-10 Tropfen täglich, bei Kindern 1 Tropfen pro die). Dieses Jodeiweisspräparat ist dem Jodkali und anderen Jodpräparaten vorzuziehen, weil es nicht schnell ausgeschieden wird und dennoch weder Jodismus noch Magenstörungen hervorruft. Energische Quecksilberkuren sind erfahrungsgemäss bei Tuberkulösen kontraindiziert und auf milde intervalläre Anwendung zu beschränken.

Viele hartnäckige Fälle von Lungentuberkulose, bei denen man bisher den Zusammenhang mit Syphilis nicht erkannt hat, werden oft durch diese Jodtherapie eine schnelle und auffallende Besserung zeigen. Der Effekt hängt naturgemäss ab von der Zeitdauer der bestehenden Erkrankung und dem Alter des Individuums. Bei jungen Individuen und in dem Stadium der entzündlichen Hypertrophie ist der Erfolg meist ein rascher und deutlicher, während man bei älteren Individuen in dem Stadium der Cirrhose weniger erreichen kann.

Nachstehend ein Fall von einer lange bestehenden Lungenerkrankung, bei der mit Jod eine schnelle Heilung erzielt wurde.

Schlossverwalter W. ist seit 26 Jahren lungenkrank. Seit einem halben Jahre mehr Husten, viel Auswurf. T.B. vom Hausarzt und hier spärlich nachgewiesen. Patient ist verheiratet und hat gesunde Kinder. Keine Syphilis angeblich durchgemacht.

15. VI. 06. Status: Linke Seite beim Atmen beinahe immobil. Ganze linke Seite Dämpfung. Überall zahlreiche mittelblasige und feine Rhonchi.

R. Spitze relat. Dämpfung, vesiko-bronchial. Atmen. Ganze rechte Seite feinere Rhonchi, Giemen und Knacken.

Sputum $20 \mathrm{ccm}$.

Temperatur febril, s. Kurve.

Verordnung: Jodtinktur in Milch, 2 mal 20 Tropfen täglich.

26. VI. 06. Nirgends mehr Rhonchi in der ganzen linken Lunge, als vereinzelte an der Basis und ein giemendes Geräusch an der Spitze

27. VIII. 06. R. Spitze rauhes Atmen.

L. Lunge vesiko-bronchial. Atmen, keine $\mathrm{Rhonchi}$.

Temperatur normiert, sputumfrei, $3 \mathrm{~kg}$ Zunahme.

1) Hergestellt von Apotheker Lang in Davos. 


\section{Klimatische Behandlung.}

Selbstrerständlich wird man da, wo man über gute klimatische Verhältnisse verfügt, diese zur Behandlung mit heranziehen. Dass die klimatische Behandlung bei der spezifischen I.K.-Therapie entbehrlich ist, beweisen die guten, zum Teil ausgezeichneten Resultate, die Ärzte in ungünstigen klimatischen und sozialen Verhältnissen mit I.K. erzielt haben: u. a. Pumr (s. u.), Lovacs, Awtokratoff (Minenarzt in Sibirien), Hollos, der ausdrücklich hervorhebt, dass bei seinen gewonnenen günstigen Resultaten die Patienten "grössenteils der ärmsten Volksklasse angehören".

Immerhin ist die Einwirkung des Hochgebirges auf die gesamte körperliche Vitalität von unverkennbarem Einfluss.

Spenglers klimatische Behandlung geht von den Grundsätzen einer vernunftgemässen und individuellen Behandlung aus. Die schematischen Liegekuren verwirft er und verfährt nach folgenden Prinzipien:

1. Nichtfiebernde Patienten beginnen die Aussenliegekur, sobald die Liegehallen durch die Sonne erwärmt werden, oder die Aussentemperatur auch ohne Sonne den Liegeaufenthalt angenehm macht.

2. Nur gute Esser dürfen bei niedriger Aussentemperatur ausgiebigen Gebrauch von der Liegekur im Freien machen, weil bei den andern der Kalorienverbrauch durch die Kälte zu stark in Anspruch genommen wird, so stark, dass er durch die Nahrungsaufnahme eventuell nicht ausgeglichen werden kann. Dadurch erklärt sich manche unverständliche Gewichtsabnahme. Forzierte Liegekur in der Kälte kann Inanitionszustände provozieren, die progressiver Phthise ähneln.

3. Aussen-Liegekur ist immer untersagt, weil schädlich, wenn der Patient im Freien friert, oder sich nur unbehaglich fühlt.

4. Nach dem Mittagessen ist 2stündige Liegekur im Bett oder in der Sonne Vorschrift.

Die körperlichen Leistungen des Patienten müssen natürlich seiner Konstitution angemessen werden. Gewöhnlich lassen wir die Patienten vormittags und nachmittags je $1 / 2-1$ Stunde spazieren gehen; zunächst nur auf ebenen Wegen. Im Verlauf der Heilung bezw. Besserung können diese Spaziergänge verlängert werden, später kommt auch leichtes Steigen hinzu. Gegen eine Erschlaffung der Muskulatur ist Massage empfehlenswert und oft bei Bettlägerigen von grossem Nutzen.

Auf die eigentümliche, bis jetzt von den Spezialärzten kaum gewürdigte Tatsache der Bewegungsentfieberung vereinzelter Tuberkulöser muss hier noch hingewiesen werden. Die meisten, auch nur leicht fiebernden Phthisiker fühlen sich bei ausgiebiger Ruhebehand- 
lung am wohlsten und entfiebern auch am leichtesten, weun man sie nicht gehen lässt.

Ein kleiner Prozentsatz der leicht febrilen Fälle verhält sich entgegengesetzt: sie sind bei strenger Ruhetherapie schwer, bei Einschaltung schonender Bewegung leicht zu entfiebern. Und das subjektive Befinden ist bei Vornahme von Gehübungen erheblich besser.

Die Ursache dieses abweichenden Verhaltens ist nicht genügend erforscht. Sehr wahrscheinlich ist der günstige Einfluss der Bewegung auf eine Autotuberkulinisierung zurückzuführen, die bei diesen Formen von Erkrankung nur durch Bewegung erreichbar ist.

Die nach der Spenglerschen Klassen-Stadien-Einteilung unter Stadium $3 \mathrm{~b}$ einrangierten Fälle sind solche schwerster Art, und unter diesen auch solche, bei denen infolge der Starrwandigkeit der Kavernen oder sonstiger anatomischer Veränderungen in den Lungen eine spontane Heilung für gewöhnlich nicht erfolgt, sondern nur durch operative Eingriffe zu erreichen ist. In einem Teil der Fälle ist die Heilung nur durch die Thorakoplastik, wie sie Carl Spengler zuerst angegeben hat und Prof. Brauer mit grossem Erfolg ausführt, erreichbar. Für einen andern Teil der Fälle kommt der Pneumothorax in Betracht. Hier hat Brauer mit seinen Arbeiten über Pneumothorax der Tuberkulose-Therapie hervorragende Dienste geleistet. Die Ausführung des Pneumothorax nach seinen und Forlanin is Angaben kann in vielen schweren Fällen, die sonst unheilbar sind, noch eine Heilung erzielen. Ich möchte an dieser Stelle die Anregung dazu geben, beide operative Eingriffe, Thorakoplastik sowohl wie Pneumothorax, mit der I.K.-Therapie zu kombinieren. Die Immunisierung, vor der Operation vorgenommen, wird günstigere anatomische Verhältnisse für dieselbe schaffen, und $n a c h$ der Operation wird sie eine schnellere Heilung ermöglichen.

\section{Literatur.}

1. Carl Spengler, Tuberkulose-Immunblut, Tuberkulose-Immunität und Tuberkulose-Immunblut- (I.K.) Behandlung. Deutsche med. Wochenschr. 1908. Nr. 38.

2. Masing, Über Bronchophonie der Flüsterstimme. Beitr. z. Klin. d. Tuberk. Bd, VII. 1907.

3. Moses, Über die Auskultation der Flüsterstimme. Beitr. z. Klin. d. Tuberk. Bd. IV. 1905. 
4. Gidionsen, Über Fieber und Fiebermessung. Beitr. z. Klin. d. Tuberk. Bd. IV. 1905.

5. Armando Gião, Phthisiodiagnostik und Therapie Dr. C. Spenglers in Davos. "Polytechnia“. Vol. III. Nr. 2. 1906. Lissabon.

6. C. Spengler, Neue Färbemethoden für Perlsucht- und Tuberkelbazillen und deren Differentialdiagnose. Deutsche med. Wochenschr. 1907. Nr. 9.

7. Brauns, Die Grundprinzipien des C. Spenglerschen Tuberkulose-, Schutzund Heilimpfverfahrens. Bericht über die IV. Versammlung der TuberkuloseÄrzte. 1907.

8. Kürthi, Die Differentialfärbemethoden der Tuberkulose-Eureger. Aus dem Privatlaboratorium des Dr. C. Spengler in Davos. Wien. klin. Wochenschr. 1907. Nr. 49.

9. v. Betegh, Neue differentialdiagnostische Färbemethode für Tuberkel-, Perlsucht- und andere säurefeste Bazillen, nebst Strukturstudien bei verschiedenen säurefesten Bakterienarten. (Aus dem Laboratorium des Dr. C. Spengler in Davos.) Zentralbl. f. Bakteriologie. Bd. XLVII. Heft 3.

10. C. Spengler, Zur Diagnose und Prognose der Misch- und Begleitinfektion bei Lungentuberkulose. Zentralbl. f. Bakteriol. XXX. Bd. 1901. Nr. 20.

11. S. Fuchs-Wolfring, Zur Carl Spenglerschen Blutzellenimmunität.

12. C. Spengler, Zur Diagnose geschlossener Lungentuberkulose der Sekundärinfektion, tuberkulöser und syphilitischer Phthise. Davos 1900.

13. C. Spengler, Die Erbdisposition in der Phthiseentstehung, ihre Diagnose und Behandlung. Deutsche med. Woch. 1906. Nr. 15.

14. S. Klein, Tuberculose et Syphilis, étudiées dans un milieu spécial. (Infirmerie spéciale de St. Lazare). Thèse de Paris. 1909.

15. Hollos, Die tuberkulösen lntoxikationen. Vortrag auf dem Internat. Kongress Budapest 1909, und Franklin-társulat, Budapest 1909.

16. A w tokrat off, Die Behandlung der Tuberknlose durch Carl Spenglersche Immunkörper (I. K.) in der Dorfambulanz. Wratschebnaja Gazeta (russ.) Nr. 49 u. 50. 1909. 


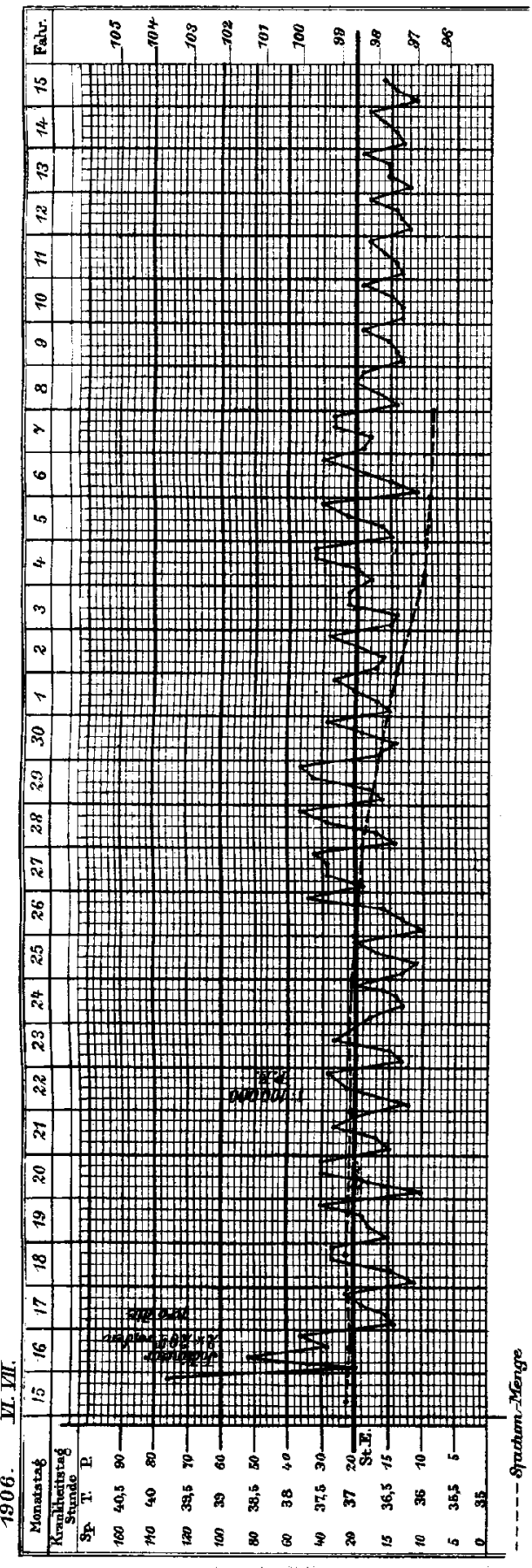

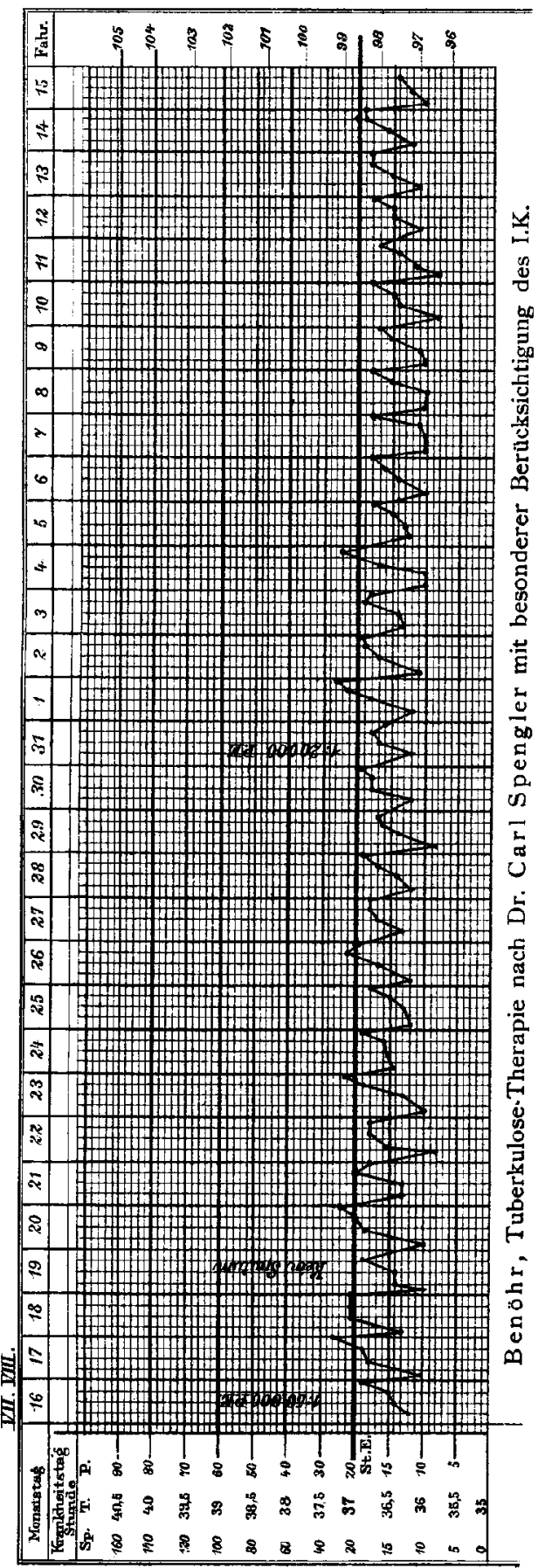

